\title{
Atomic imaging in aberration-corrected HRTEM with application to $\mathrm{Al}$ alloys
}

\author{
J. H. Chen ${ }^{1,2}$, K. Urban ${ }^{2}$, B. Kabius ${ }^{2}$, M. Lentzen ${ }^{2}$, J. Jansen ${ }^{1}$ and H. W. Zandbergen ${ }^{1}$ \\ 1 National Center for HREM, Delft University of Technology and Netherlands Institute for Metals \\ Research, Rotterdamseweg 137, 2628 AL Delft, The Netherlands \\ 2 Institute for Solid State, Research Center Juelich, D-52425 Juelich, Germany
}

Since the successful correction of the spherical aberration (Cs) of the objective lens in a Philips CM 200 FEG ST microscope achieved by Rose and Haider et al [1-2], a few theoretical and computational expectations have been made to show the possible atomic imaging conditions in the Cs-corrected high-resolution transmission electron microscopy (HRTEM) [3-4]. These conditions include the phase-contrast imaging at a small defocus, the amplitude-contrast imaging at about zero defocus and the projected charge density (PCD) contrast imaging at a small over-focus. Meanwhile still some controversial opinions do exist concerning whether or not through-focus exit-wave function reconstruction (EWR) [5] and Cs-corrected HRTEM are complementary or exclusive. Up till now no systematic experimental and theoretical investigations on these issues have been carried out and therefore the theories about the optimum imaging conditions and the image interpretation remain to be either justified or established.

In the present work, we conducted a systematic experimental investigation on the image regime of the new microscope, aiming to achieve the highest resolution directly in interpretable atomic images. Atomic images of a $0.136 \mathrm{~nm}$ resolution, which was the measured information limit of the microscope, have been obtained in different imaging ways. We also performed through-focus EWR, showing that in this microscope a posteriori correction of the exit-wave for aberrations is greatly simplified and therefore it is a microscope very convenient for users to reconstruct the exit-wave functions. Using the advantages of the Cs-corrected HRTEM, structural details of some solute atomic clusters in an $\mathrm{Al}-\mathrm{Mg}-\mathrm{Si}-\mathrm{Cu}$ alloy system were successfully studied.

In this microscope, a double hexapole corrector system is implemented to compensate the Cs of the objective lens and at the same time to suppress all the other aberration coefficients of whole the resulting system to the values below their maxima allowed. Owing to its special structure, the microscope requires an additional alignment procedure besides the basic alignment of the microscope. After the proper alignment, the microscope has a Cs value close to zero. We have taken through-focus series of high-resolution images from $\mathrm{Si}[110]$ specimens for varying specimen thickness. The obtained images were analyzed with image simulation and image processing.

In this novel TEM we are able to obtain $\mathrm{Si}[110]$ atomic images of $0.136 \mathrm{~nm}$ resolution in different imaging regimes. For conventional phase-contrast imaging, silicon black atom dumbbells are observed. For the amplitude-contrast, or more accurately, channeling-contrast imaging, atomic column positions in the images are indicated by the intensity maxima due to the electron channeling effect along columns. In the wave function reconstructed from through-focus image series taken from very thin specimens, the phases are linearly proportional to the projected potentials of atomic columns. Some of these ultra-high resolution images are shown in Fig.1a-d. It is also shown that a 
high signal-to-noise ratio and therefore proper image processing techniques are particularly important for the new microscope to achieve its highest resolution [6].

\section{References}

[1] H. Rose, Optik 85 (1990), 19.

[2] M. Haider, et al, Nature, Vol.392 (1998), 768.

[3] M. Foschepoth and H. Kohl, Phys. Stat. Sol. (a) (1998), 357.

[4] M. A. OKeefe, Proc. 14th Int. Congr. for Electr. Micros. (Cancun, Mexico 1998), Vol.1, p.163.

[5] W. Coene, et al., Ultramicroscopy 64 (1996), 109.

[6] This work was supported by German Science Foundation (DFG) and Netherlands Institute for Metals Research (NIMR).

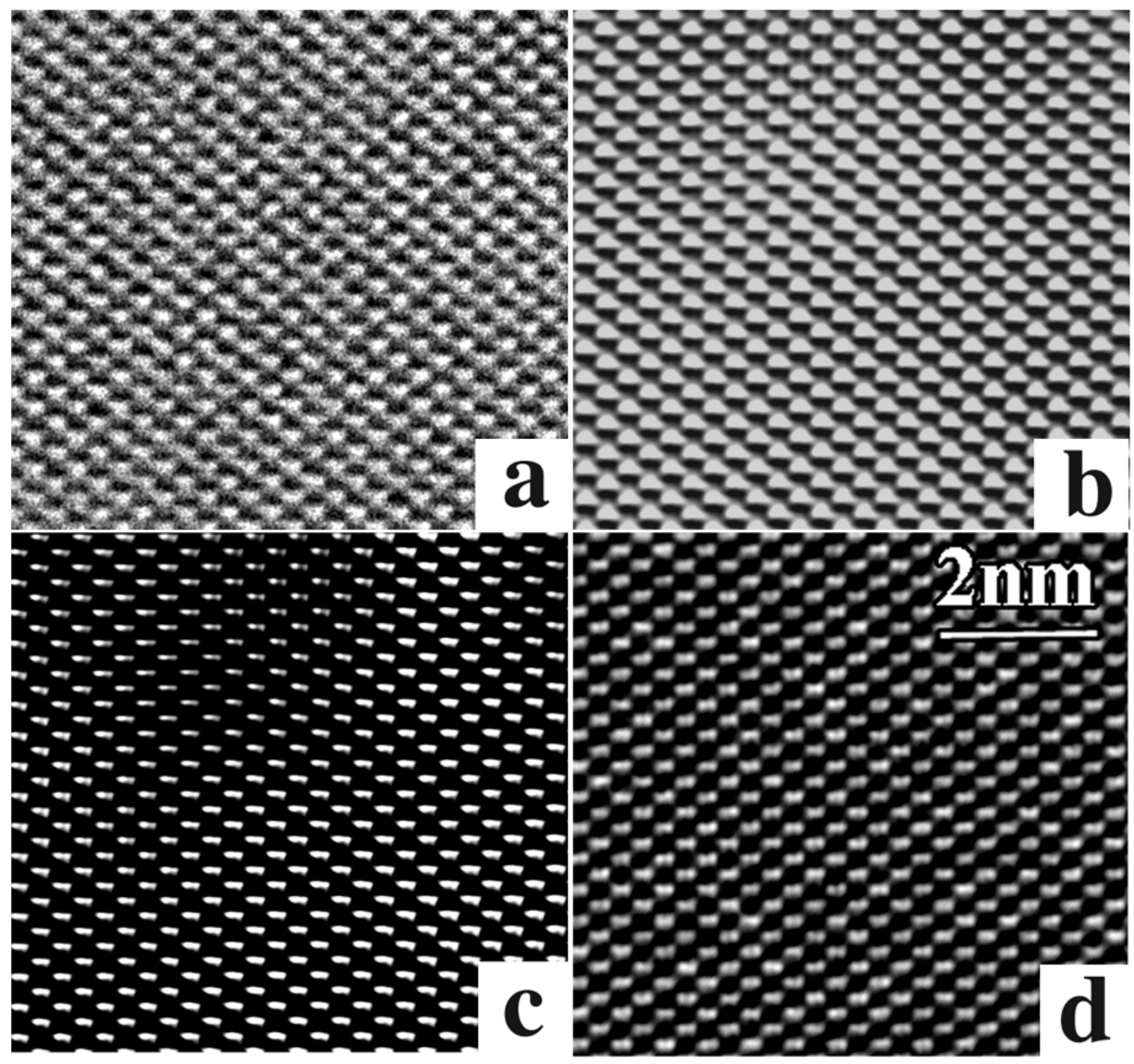

Figure.1 (a) original phase-contrast image recorded at $-7 \mathrm{~nm}$ defocus for $\mathrm{Si}[110]$, showing that the noise nearly destroys the black atom dumbbells. (b) Fourier-filtered image of (a) for a reduction of the noise. (c) inverted image of (b) for a better visualization of the atom dumbbells. (d) phase-image of the exit-wave function reconstructed from 20 Cs-corrected HRTEM images for Si[110]. 